\title{
Bemerkungen zum Altpleistozän des Illergletscher-Gebietes *)
}

\author{
Karl Albert HabBe**)
}

\begin{abstract}
Lower Pleistocene, Mindel glaciation, Haslach glaciation, Middle Pleistocene, Riß glaciation, glaciofluvial features (Older Deckenschotter, Younger Deckenschotter), moraines, extent, geomorphological sketch maps

German Alpine Foreland (Iller area), Bavaria
\end{abstract}

TK 25: Nr. 8127

\begin{abstract}
Kurzfassung: Die Geomorphologische Kartierung des Blattes 8127 Grönenbach machte eine Auseinandersetzung mit den divergierenden Ergebnissen neuerer Untersuchungen zum Altpleistozän des Illergletscher-Gebietes notwendig. Es ergab sich, daß die im Blattgebiet auftretenden altpleistozänen glazifluvialen Bildungen (Ältere und Jüngere Deckenschotter) als im wesentlichen einheitliche, zeitlich nicht zu untergliedernde Schüttungen zu betrachten, und daß die Altmoränen eindeutig in drei Komplexe zu gliedern sind, die der Riß-Eiszeit, der Mindel-Eiszeit sensu PENCK 1901 ( „klassisches” Mindel) und einer weiteren, zwischen klassischem Mindel und Günz einzuordnenden Eiszeit (möglicherweise der Haslach-Eiszeit sensu SCHREINER 1981) zugewiesen werden.
\end{abstract}

[Remarks on Deposits and Relief Forms of the Older Pleistocene of the Iller Glacier Area (Bavarian Alpine Foreland)]

Abstract: Geomorphological mapping of sheet 8127 Grönenbach made it necessary, to discuss the diverging findings of some recent studies on the Older Pleistocene of the former Iller glacier. The result is, that the glaciofluvial features of the Older Pleistocene in the area (Older Deckenschotter, Younger Deckenschotter) have been deposited as essentially uniform accretions, and that the Older Moraines can be clearly subdivided into three moraine complexes, which are assigned to the Riss glaciation, the Mindel glaciation sensu PENCK 1901 ('classical' Mindel) and a still older glaciation, which is to be intercalated between the classical Mindel and the Günz glaciation (possibly the Haslach glaciation sensu SCHREINER 1981).

*) Überarbeitete Fassung eines Vortrags, der am 12. 9. 1985 auf der 22. Wissenschaftlichen Tagung der Deutschen Quartärvereinigung in Freiburg gehalten wurde. Aus technischen Gründen können hier nicht alle während des Vortrages gezeigten Abbildungen reproduziert werden. Sie sind in den Originalveröffentlichungen (PENCK \& BRÜCKNER 1901/09; EBERL 1930; SINN 1972; SCHAEFER 1973; GLUUCKERT 1974; JERZ et al. 1975; STEPP 1981) nachzuschlagen.

**) Anschrift des Autors: Prof. Dr. K. A. HabBE, Institut für Geographie der Universität, Kochstr. 4, 8520 Erlangen.

\section{Vorbemerkungen}

Das Gebiet des pleistozänen Iller-Gletschers und seines glazifluvialen Vorfelds - der Iller-Lech-Platte ist bekanntlich die Typregion für A. PENCKs Viergliederung der alpinen Eiszeiten (PENCK \& BRÜCKNER 1901/09) gewesen und hat auch nach PENCK immer wieder - für die Stratigraphie des Pleistozäns allgemein wichtige - neue Ergebnisse geliefert. Erinnert sei nur an die Untersuchungen von B. EBERL (1930), an I. SCHAEFERs und H. GRAULs Arbeiten aus den 40er und 50er Jahren (SCHAEFER 1940; GRAUL \& SCHAEFER 1953), an die Untersuchungen der Heidelberger Graul-Schüler aus den 70er Jahren (SINN 1972; EICHLER \& SINN 1975; LÖSCHER 1976) und an die daraus erwachsene - ja noch keineswegs abgeschlossene Diskussion mit I. SCHAEFER.

Vergleicht man jedoch das Kärtchen der „Schotterfelder der Gegend von Memmingen” aus den „Alpen im Eiszeitalter" (PENCK \& BRÜCKNER 1901/09: 29, Fig. 2, - die gleiche Abbildung auch bei SCHAEFER 1973: 169, Abb. 1), an dem PENCK sein tetraglaziales System demonstriert hatte, mit der Geologischen Übersichtskarte des Iller-Mindel-Gebiets 1:100000 von H. JERZ et al. von 1975 (die Karte beruht für das in Frage stehende Gebiet weitgehend auf SINN 1972 und GLÜCKERT 1974 und war ihrerseits Grundlage für das von H. SCHOLZ und W. ZACHER bearbeitete Blatt CC 8726 Kempten der Geologischen Übersichtskarte $1: 200000$ von 1983), dann scheint es auf den ersten Blick in diesem engeren Illergletscher-Vorfeld in einem Dreivierteljahrhundert kaum zu neuen Erkenntnissen gekommen zu sein: dem Memminger Feld PENCKs entspricht nach wie vor die Niederterrasse, seinem Hitzenhofener Feld die Hochterrasse, dem Grönenbacher Feld die Jüngeren Deckenschotter und dem PENCKschen Hochfeld die Älteren Deckenschotter (vgl. dazu Abb. 1 u. 2). 


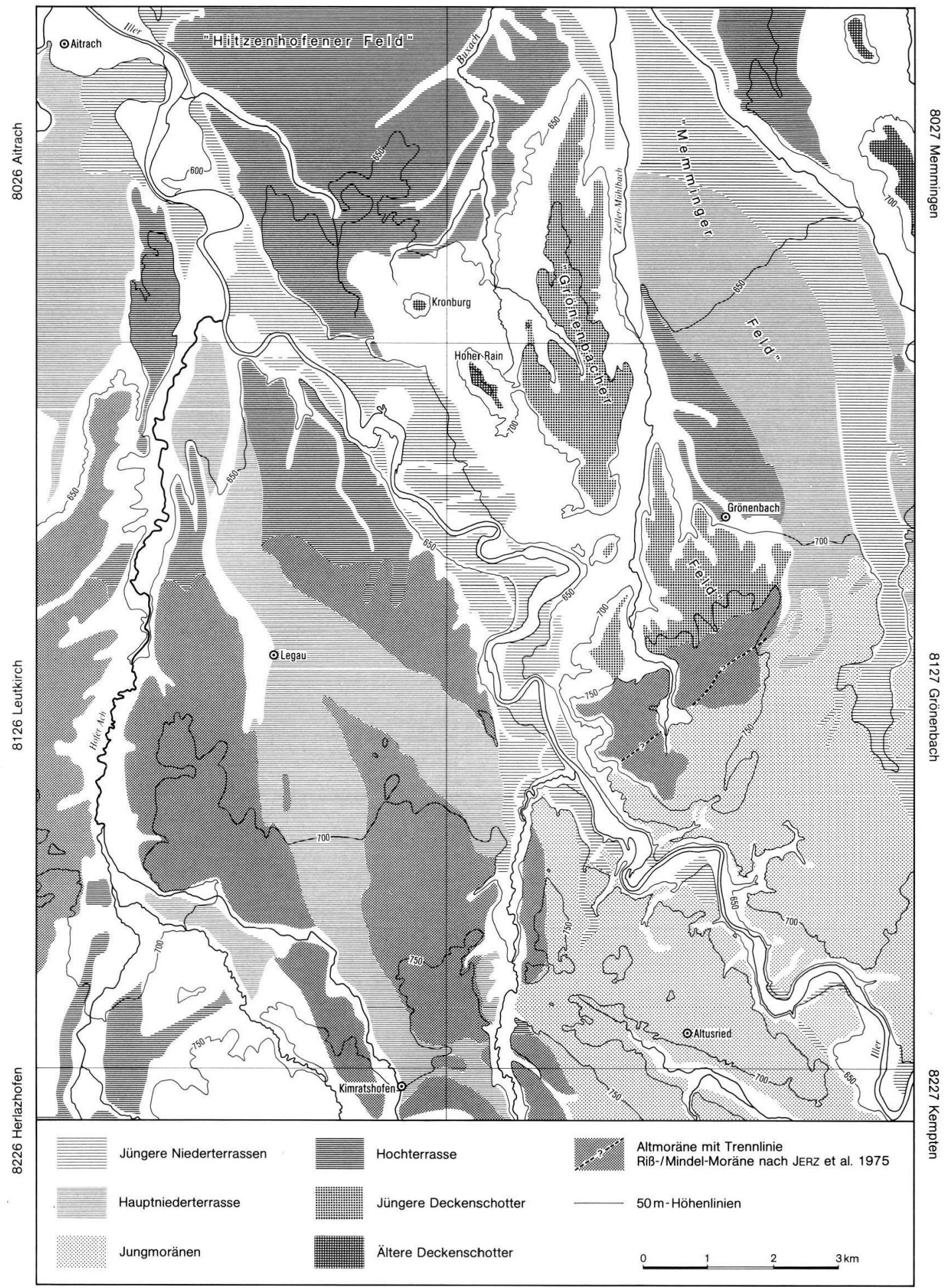

Abb. 1: Geologisch-morphographische Übersichtskarte von Blatt Grönenbach und seinen Randgebieten (Westabschnitt). 


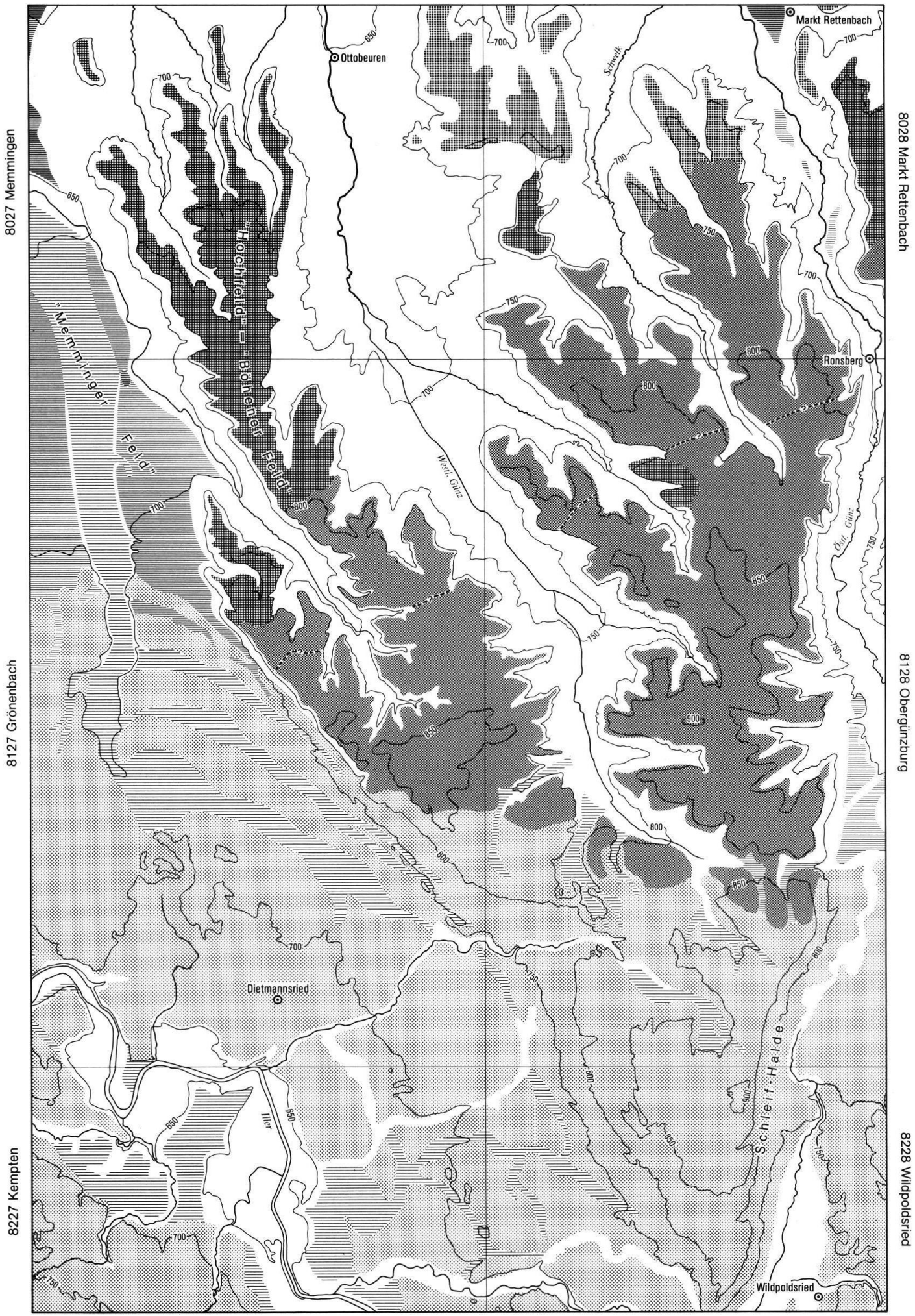

Abb. 2: Geologisch-morphographische Übersichtskarte von Blatt Grönenbach und seinen Randgebieten (Ostabschnitt). 
Dieser erste Eindruck täuscht jedoch. Einmal zeigt die JERZsche Karte aus technischen Gründen nicht (was dann in den Erläuterungen aber dargelegt wird), daß sowohl Niederterrasse wie Hochterrasse je in zwei Niveaus vorliegen (was PENCK noch nicht gesehen hatte). Die JERZsche Karte berücksichtigt weiter nicht den zum Zeitpunkt ihres Erscheinens bereits vorliegenden Versuch I. SchaEFERs (1973), die PENCKsche Typlokalität für die Mindel-Eiszeit das Grönenbacher Feld - vollkommen neuzugliedern. Sie konnte schließlich nicht die Umdatierung der PENCKschen Schotterfelder durch M. LÖSCHER (1976) und R. STEPPs (1981) Versuch einer Neugliederung der Älteren Deckenschotter des Böhener Feldes berücksichtigen. Andererseits macht die JERZsche Karte dezidierte Aussagen über die Verbreitung der zu den ausgeschiedenen Schotterfeldern gehörigen Moränen, die schon PENCK eher dilatorisch behandelt hatte und die auch in den meisten späteren Arbeiten nur am Rande mitbesprochen werden. Diese Aussagen über Moränenverbreitung und -gliederung waren für den Entwurf einer geologischen Karte notwendig, sie stehen aber im Widerspruch zu den Vorstellungen älterer Autoren und erscheinen auch aus anderen Gründen problematisch. Tatsächlich liegen also auch im engeren Vorfeld des Iller-Gletschers eine ganze Reihe ungelöster quartärstratigraphischer Fragen vor.

Dieser Sachlage war Rechnung zu tragen, als die Erläuterungen zu der - im Rahmen des DFG-Schwerpunktprogramms „Geomorphologische Detailkartierung in der Bundesrepublik Deutschland" durchgeführten - geomorphologischen Kartierung des Blattes 8127 Grönenbach der Topographischen Karte 1:25000 (HABBE 1985 b) zusammengestellt wurden. Das Gebiet von Blatt Grönenbach wird zwar im S vorwiegend durch Ablagerungen und Formen der Würm-Eiszeit geprägt (vgl. dazu HABBE 1985a), umfaßt aber im $\mathrm{N}$ auch Ablagerungen der drei älteren PENCKschen Eiszeiten, über die die Diskussion noch im Gange ist. Die Kartierung erzwang hier eine Stellungnahme, obwohl ihr Zweck primär nicht die Präzisierung der Quartärstratigraphie, sondern die Erprobung eines Kartierungsschlüssels war. Die vorliegenden Beobachtungen - nicht nur im Blattgebiet, sondern auch im Bereich der Nachbarblätter - mußten entsprechend geprüft, die kontroversen Schlußfolgerungen gegeneinander abgewogen, das Ergebnis schließlich kartographisch fixiert und in den Erläuterungen begründet werden. Entscheidungskriterium war dabei in der Regel, ob und inwieweit sich die Ergebnisse der vorliegenden Publikationen in den gegebenen morphologischen Zusammenhang einpassen ließen. Die wesentlichen Ergebnisse dieser Überlegungen werden im folgenden zur Diskussion gestellt.

\section{Das Grönenbacher Feld}

Bei der Neuaufnahme des Grönenbacher Feldes hatte SCHAEFER (1973) die Tatsache, daß dessen Schotterbasen an den Flanken der das Feld durchschneidenden jüngeren Täler in unterschiedlichen Höhenlagen ausstreichen, dahingehend interpretiert, daß das Grönenbacher Feld nicht - wie das alle älteren Autoren seit PENCK getan hatten - als eine einheitliche Schüttung aufzufassen sei, sondern als eine Folge von insgesamt zehn Eintiefungs- und WiederaufschüttungsZyklen (vgl. dazu SCHAEFER 1973: 174, Abb. 3 und Tafel I). Die Mindel-Eiszeit sei entsprechend als „Großeiszeit (oder . . . Eiszeitengruppe)” (SCHAEFER 1973: 198) zu klassifizieren.

Grundannahme der SCHAEFERschen Argumentation ist, daß jeder glazifluvialen Aufschüttung eine Erosionsphase vorausgehe, die dazu führe, daß die Schotterbasis ein kastenförmiges Querprofil aufweise (SCHAEFER 1950 u. ö., zuletzt 1981). Dies ist jedoch nicht unumstritten. Es gibt heute bereits eine ganze Reihe von Untersuchungen (SCHREINER 1980; HAAG 1981, 1982; RÖGNER 1979, 1980, 1981), die anhand von Bohrungen belegen, daß ein kastenförmiges Querprofil der Schotterbasen keineswegs der Regelfall sein muß, insbesondere dann nicht, wenn die glazifluviale Verschüttung erstmals erfolgt und ein Tal betrifft, das zuvor unter periglazialen Bedingungen entstanden war. Ob die SCHAEFERschen Schlußfolgerungen zutreffen oder ob seine - an sich vorbildlich korrekt erhobenen - Beobachtungen nicht vielmehr zu Querprofilen führen müssen, wie sie schon PENCK gezeichnet hat (PENCK \& BRÜCKNER 1901/09: 31, Fig. 3, - die gleiche Abbildung bei SCHAEFER 1973: 172, Abb. 2 oben), müßte also streng genommen anhand einer Folge engstehender Bohrungen geklärt werden. Zweifel an der SCHAEFERschen Interpretation erscheinen aber jedenfalls erlaubt.

Denn schon das Oberflächenrelief des Grönenbacher Feldes stützt sie nicht (vgl. dazu die $\mathrm{OH}$-Ausgabe der TK 1 : 50000 , Blatt L 8126 Memmingen). Eine Untergliederung in schmale Terrassenstreifen ist nicht erkennbar, - wenn man einmal davon absieht, daß das Feld im Nordteil eine leichte W-E-Asymmetrie aufweist. Daß der Westflügel hier generell etwas höher liegt, läßt sich jedoch zwanglos durch die periglaziale Überformung während der der Ablagerung folgenden Kaltzeiten erklären. SCHAEFERs Vorstellungen erscheinen aber auch noch aus anderen Gründen problematisch. Sie erklären nämlich nicht (oder nur durch Zuatzhypothesen),

- weshalb die der jeweiligen Einschneidung folgende Wiederaufschüttung stets - was ganz ungewöhnlich wäre - das Ausgangsniveau gerade wieder ereicht haben sollte (vgl. dazu SCHAEFER 
1973: Tafel I, besonders Profil 7) oder - falls das nicht der Fall war - weshalb dann jüngere Wasserläufe wie der östlichste Zubringer der Buxach (vgl. dazu Abb. 1 und SCHAEFER 1973: $174, \mathrm{Abb} .3$ ) von jüngeren Schotterfüllungen (in diesem Fall dem ScHAEFERschen Schotter VII) her auf ältere Schotter (hier die SCHAEFERschen Schotter VI und V) mit höherliegender Aufschüttungsoberfläche hätten übertreten und diese zerschneiden können sollen,

- weshalb die angenommenen Vorstöße der mindelzeitlichen Gletscher ihre Schmelzwasserrinnen zwar ständig tiefergelegt, sie dabei aber nicht wie es normal gewesen wäre - konzentriert, sondern zunehmend - in schließlich vier Rinnen (SCHAEFER 1973; 193, Abb. 4) - aufgesplittert haben sollen,

- wie jüngere Schotter, die nach rückwärts keine Verbindung zum Schmelzwasserursprung an den zugehörigen Gletscherstirnen haben, unvermittelt zwischen älteren Schottern auftauchen können (Schotter X zwischen Schotter VI und VII westlich Grönenbach und zwischen Schotter VIII und IX westlich Waldegg, - vgl. dazu SCHAEFER 1974: 174, Abb. 3),

- weshalb die den Schotterkomplex im S abschließenden mindelzeitlichen Endmoränen am oberen Ende der jüngsten und am stärksten eingetieften Rinnen (Schotter IX westlich Waldegg, - vgl. SCHAEFER 1973: 174: Abb. 3) keine Schmelzwasserdurchlässe aufweisen, sondern deren Füllungen eindeutig überlagern.

Zudem ist an keiner Stelle nachgewiesen, daß die von SCHAEFER unterschiedenen Schotterstränge tatsächlich so, wie es seine Profile zeigen, durch steil einfallende Erosionsdiskordanzen voneinander getrennt sind.

Diese Unstimmigkeiten lassen sich beheben, wenn man nicht - wie SCHAEFER - davon ausgeht, daß die Bildung des Grönenbacher Feldes bereits mit dem Beginn der Mindel-Eiszeit eingesetzt habe. Der aus dem der Mindel-Eiszeit vorhergehenden Interglazial überkommene Abfluß des Iller-Gletschers (also die Ur-Iller) muß nämlich noch zu Beginn der MindelEiszeit aus der Gegend von Kempten nach NNE gegen das heutige Mindel-Tal gezielt haben. Für die Günz-Eiszeit ist diese alte Abflußrichtung seit EBERL (1930) belegt. Aber auch in den nachfolgenden Kaltzeiten sind die glazifluvialen Schüttungen der vorstoßenden Gletscher immer zunächst in NNE-Richtung erfolgt. Das beweisen der ausgedehnte und mächtige Jüngere Deckenschotter von Oberegg-Saulengrain südsüdwestlich Mindelheim und der ihm westlich benachbarte, etwas tiefer liegende, aus dem Östlichen Günz- ins Mindel-Tal hinüberwechselnde Jüngere
Deckenschotter von Unterburg-Erlis (vgl. dazu die JeRZsche Karte und SinN 1972: 93 ff.). Beide Schotter setzen trotz - bezogen auf den Austritt des IllerGletschers aus dem Gebirge südlich Kempten wesentlich externerer Lage höher an als das Grönenbacher Feld. Sie hätten nicht geschüttet werden können, wenn der tieferliegende Schmelzwasserabfluß über das Grönenbacher Feld zur Zeit ihrer Entstehung schon existiert hätte. Das Grönenbacher Feld muß also später entstanden sein. Wahrscheinlich ist, $\mathrm{da}$ es auf die gleiche Weise zustandegekommen ist, die sich für die verschiedenen Abflußrinnen des würmzeitlichen wie des rißzeitlichen Iller-Gletschers im Detail nachweisen läßt: nämlich erst kurz vor dem Hochstand des mindelzeitlichen Gletschers durch Schmelzwasserüberläufe in ein benachbartes autochthones Talsystem. Dafür spricht neben der tiefen Lage des Grönenbacher Feldes (und seiner Fortsetzung im $\mathrm{N}$ bei Schwaighausen) das auffallende Ausheben seiner Schotterbasis gegen deren Wurzeln hin (vgl. dazu SINN 1972: Längsprofil 5 und SCHAEFER 1973: Tafel II).

Das Grönenbacher Feld ist danach als eine relativ rasch geschüttete einheitliche Bildung aufzufassen, was auch den recht gleichförmigen Schotterbestand erklären würde. In der Schlußphase der Entwicklung ist dann der mindelzeitliche Gletscher auf seine eigenen Schotter aufgefahren und hat die das Feld im S abschließenden Moränen abgelagert, - so wie das der rißzeitliche Gletscher beim Hitzenhofener Feld und der würmzeitliche Gletscher beim Memminger Feld auch getan haben. Im Prinzip hat diesen Zusammenhang bereits SINN (1972: 105 f.) dargestellt. Unbeschadet einer zukünftigen Detailuntersuchung der Schotterbasen darf also weiterhin angenommen werden, daß das Grönenbacher Feld - so wie das auch alle älteren Autoren seit PENCK gesehen haben - als eine einheitliche Bildung eines einzigen GletscherGroßvorstoßes, eben der Mindel-Eiszeit sensu PENCK $\mathrm{zu}$ betrachten ist. Entsprechend kann das - in die Achse des Memminger Trockentals projizierte Längsprofil des Grönenbacher Feldes weiter so gezeichnet werden, wie es schon PENCK (PENCK \& BRÜCKNER 1901/09: Tafel I, Profil 1) getan hat (vgl. dazu Abb. 3).

\section{Das Böhener Feld}

Für den Älteren Deckenschotter des von STEPP (1981) sogenannten Böhener Feldes - PENCKs „Hochfeld” also bzw. den Schrattenbach-Theinselberger Schotter sensu SINN (1972) - gilt ähnliches. STEPP hatte ihn aufgrund der Schotterunterkanten-Ausstriche in sechs Schotterstränge gegliedert (STEPP 1981: 51, Abb. 4 und 64, Abb. 8). Dagegen können die gleichen 


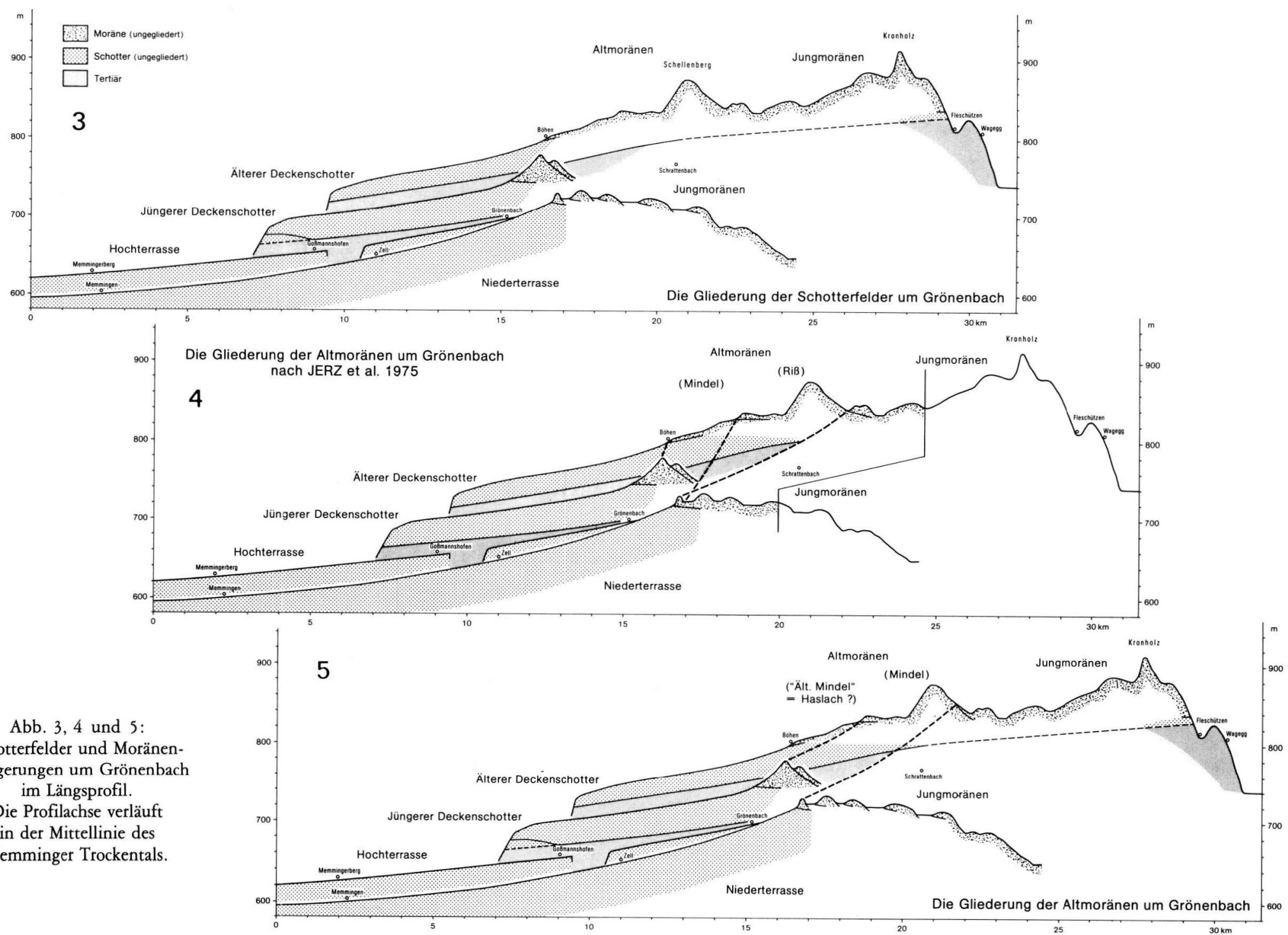

Schotterfelder und Moränenablagerungen um Grönenbach im Längsprofil.

Die Profilachse verläuft in der Mittellinie des Memminger Trockentals. 
Bedenken vorgebracht werden wie gegen die SCHAEFERsche Interpretation des Grönenbacher Feldes.

Zunächst stützt auch hier das Oberflächenrelief (vgl. dazu die $\mathrm{OH}$-Ausgabe der TK 1:50000, Blätter L 8126 Memmingen, L 8128 Kaufbeuren und L 8328 Marktoberdorf) die STEPPschen Annahmen nicht: das Böhener Feld weist eine ausgesprochen gleichmäßige Abdachung nach $\mathrm{N}$ auf, die eine Gliederung in Teilstränge nicht erkennen läßt. Gegen separat eingetiefte und bis auf ein einheitliches Oberflächenniveau je wiederaufgefüllte Rinnen spricht hier wie beim Grönenbacher Feld die nicht beantwortete Frage nach der Mechanik eines solchen Vorgangs. Die kleinen Unterschiede hinsichtlich der Ausstriche der Schotterunterkanten in den Querprofilen, auch das - vor allem im Südabschnitt feststellbare - Ausheben der Schotterbasis gegen E läßt sich mit der Verschüttung eines periglazial vorgeformten Reliefs zwanglos erklären (vgl. dazu SiNN 1972: 68 ff.). STEPPs Feststellung (1981: 50) „Einen umfassenden „SchrattenbachTheinselberg-Schotter" . . ., der in Muldentälern abgelagert sei . .., gibt es nicht, denn die Querprofile $(1-8)$ zeigen ebene Sohlenflächen der einzelnen Schotterrinnen" gilt allenfalls dann, wenn die Querprofile tatsächlich so zu zeichnen wären wie STEPP es getan hat. Daran kann man aber zweifeln. STEPP selbst hat sicher nicht ohne Grund darauf verzichtet, in seine Querprofile die steil einfallenden Erosionsdiskordanzen einzutragen, die zwischen den von ihm unterschiedenen Schottersträngen zu fordern wären (zwischen den Schottern B 3 und F3 in Profil 2 sowie zwischen B 2 und E in Profil 6, - vgl. STEPP 1981: 51, Abb. 4). Im Gelände sind sie tatsächlich nirgends zu beobachten. Damit stellt sich aber die Frage, ob die Ausstriche der Schotterunterkanten in den genannten Profilen nicht direkt zu verbinden sind, und damit das gleiche Problem, das schon beim Grönenbacher Feld angeschnitten wurde.

Im übrigen hat sich STEPP hinsichtlich der zeitlichen Stellung der von ihm unterschiedenen Schotterstränge zueinander nicht näher geäußert. Geht man von seinen Profilen aus, müßte der Schotter $C$ wegen seiner generell höhergelegenen Basis der älteste sein, der Schotter F mit der (zumeist) tiefstgelegenen Basis der jüngste (STEPP 1981: 49, Abb. 3 und 51, Abb. 4). Bei diesem nur im $\mathrm{N}$ - eingeklemmt zwischen ältere Schotter - auftretenden Schotter F läßt STEPPs Übersichtskarte (1981: 64, Abb. 8) aber die Frage offen, wie hier der Schmelzwasserzustrom von dem weit im S gelegenen Gletscherrand her erfolgt sein soll. Das gleiche gilt für die nur kleinflächig auftretenden Schotter D und E. Ferner zeigen STEPPs Längsprofile (1981: 47, Abb. 2 und 49, Abb. 3) bei den beiden westlichen Hauptschottersträngen A und B mehrere Gefällsbrüche und Profilkreuzungen, die Schotter B jünger erscheinen lassen als Schotter A, zugleich aber für Schotter B eine höher (und externer) gelegene „obere Erosionsbasis" voraussetzen. Der zugehörige Gletscher müßte danach in einer schmalen Zunge bis nördlich Schrattenbach vorgestoßen sein, wofür aber jegliche sonstige Hinweise fehlen.

Andererseits weisen alle angenommenen Rinnen des Böhener Feldes einen nach Korngröße und Gesteinsanteilen sehr einheitlichen Schotterbestand auf. Man muß daher wohl - wägt man alle bisherigen Beobachtungen $\mathrm{ab}$ - vorbehaltlich einer näheren Untersuchung der Schotterbasen durch Bohrungen annehmen, daß auch das Böhener Feld eine im wesentlichen einheitliche Schüttung ist, die - wie das jüngere Grönenbacher Feld - als Folge von Schmelzwasserüberläufen über die linke Flanke der Gletscherumrahmung in ein periglazial vorgeformtes autochthones Talsystem zustandegekommen ist, - so wie das SINN (1972: 77 ff.) in den Grundzügen schon dargestellt hat.

STEPP selbst hat dazu mit seinen Überlegungen zum „Ursprung des Böhener Feldes” (1981: 60 ff.) ein entscheidendes Argument geliefert: er konnte zeigen, $\mathrm{da} ß$ eine Beziehung zwischen den - zuerst von EBERL (1930: 298 ff.) als Äquivalent der Älteren Deckenschotter der Günztalzunge des Iller-Gletschers beschriebenen - altquartären Ablagerungen der Schleifhalde an der Westflanke des Wildpoldsrieder Zweigbeckens (zur Lage vgl. Abb. 2) und den Älteren Deckenschottern des Böhener Feldes - genauer: zum Schotter C - nur über eine in höher aufragendes Tertiär eingesenkte Rinne nördlich des FleschützenTobels hergestellt werden kann (STEPP 1981: 60, Abb. 6), die Wurzeln seiner Schotter A und B seien dagegen südlich der Schleifhalde zu suchen.

An dieser Beobachtung ist zunächst interessant, daß die von STEPP identifizierte, nach $\mathrm{N}$ gerichtete Überlaufrinne ein muldenförmiges Querprofil aufweist und eben nicht das für die Basis der Älteren Deckenschotter sonst unterstellte Kastenprofil. Wichtig ist aber außerdem, daß die Rinnenfüllung nur geschüttet werden konnte, wenn es an der Stirn des zugehörigen Gletschers weiter westlich oder südwestlich keine wesentlich tiefergelegenen Abflüsse gab. Auch die von STEPP wohl mit Recht im S der Schleifhalde vermuteten Quellpunkte der Zuflüsse zu seinen Schottern A und B (und auch zu den westlich des Memminger Trockentals gelegenen Resten Älterer Deckenschotter auf dem Hohen Rain und der Kronburg, vgl. Abb. 1) können daher nur aus vergleichbar hochgelegener Position gekommen sein. Das spricht gegen eine weiter im W gelegene breite günzzeitliche Schotterwurzelzone - wie sie STEPP annimmt - und verstärkt das zuerst von SINN (1972) vorgetragene 
Argument, daß sich im Bereich des heutigen Dietmannsrieder Zweigbeckens während der Schüttung der Älteren Deckenschotter noch eine von hochaufragendem Tertiär gebildete Wasserscheide befand, die zunächst nur über einzelne Pässe hinweg von Schmelzwasserabflüssen überschritten werden konnte. Es legt im übrigen auch die Vermutung nahe, daß es sich bei den auffälligen Erhebungen im Wasserscheidenbereich zwischen Memminger Trockental und Östlicher Günz (vgl. dazu Abb. 3-5), die die höchsten Moränenvorkommen des ganzen Alpenvorlandes tragen (Kronholz 916 m, Simmerberg 901 m, Schellenberg $877 \mathrm{~m}$ ) tatsächlich nicht um Endmoränen handelt, sondern um lediglich moränenüberkleidete Reste dieser Altwasserscheide.

\section{Die Altmoränen}

Im Gegensatz zu den altpleistozänen Schottern stellen die Altmoränen des Illergletscher-Vorfeldes ein zwar altbekanntes, aber wenig bearbeitetes Problem dar. Schon PENCK (PENCK \& BRÜCKNER 1901/09: $198 \mathrm{ff}$. und 177, Fig. 37, - zur Lage vgl. Abb. 1 u. 2) hatte aufgrund des Zusammenhangs mit entsprechenden Schottern festgestellt, daß vor den Jungendmoränen des letztkaltzeitlichen Iller-Gletschers ganz verschiedene ältere Moränen liegen: im N - in der Fortsetzung des Dietmannsrieder Zweigbeckens - nur der schmale Gürtel der Mindel-Endmoränen an der Wurzel des Grönenbacher Feldes, im NW vor dem Altusrieder Zweigbecken die relativ breite Zone der RißEndmoränen an der Wurzel des Hitzenhofener Feldes, und in dem Zwickel zwischen Dietmannsrieder und Wildpoldsrieder Becken im NE - über Älteren Deckenschottern - ein nochmals breiterer Gürtel von Altmoränen, die PENCK ganz überwiegend der Mindel-Eiszeit zuwies. Insbesondere diese hochgelegene, auf Blatt Grönenbach bereits etwa 6, weiter im $\mathrm{E}$ dann bis zu $12 \mathrm{~km}$ breite Altmoränenzone hat stets Interpretationsschwierigkeiten bereitet. Wie sie zu gliedern sei, darüber hat jeder damit befaßte Autor eigene Ansichten entwickelt (vgl. Abb. 6 und 7). Einig war man sich eigentlich immer nur über den ungefähren Verlauf der Jungmoränengrenze und darüber, daß irgendwie zwischen Riß- und MindelMoränen zu gliedern sei. Schon über die AltmoränenAußengrenze gab es stets Differenzen. Der Grund für diese offensichtlichen Unsicherheiten ist, daß Riß-und Mindel-Moränen lithologisch nicht zu unterscheiden sind und beide - ebenso wie die angrenzenden Deckenschotter - unterschiedslos von mehrere Meter mächtigen, periglazial überformten Verwitterungsdecken überlagert werden, Aufschlüsse zudem weitgehend fehlen und auch das Relief - wegen der starken jungen Zertalung des Gebietes - nur wenige Anhaltspunkte hergibt.
H. JERZ (in JERZ et al. 1975) hat deswegen seiner Abgrenzung von Riß- und Mindel-Moränen eine eigens auf das Problem angesetzte Untersuchung von G. GLÜCKERT (1974) zugrundegelegt. GLƯCKERT seinerseits hat im wesentlichen nach dem Relief gegliedert und für die Trennung von Mindel- und Riß-Moränen den von SINN (1972: $115 \mathrm{ff}$.) bekanntgemachten sogenannten Boden von Hinterschmalholz herangezogen. Das ist eine bis zu $8 \mathrm{~m}$ mächtige, periglazial überformte und daher wohl überwiegend als Bodensediment (vgl. dazu auch STEPP 1981: 56 f.) anzusprechende Bildung auf Geschiebelehm, die ihrerseits durch einen Geschiebelehm überlagert wird. Aufgrund ihrer Mächtigkeit hatte SINN sie als Bildung des Mindel-Riß- (also des ,großen”) Interglazials angesehen, die liegende Moräne entsprechend dem Mindel, die hangende dem Riß zugeordnet. Daraus ergab sich für SINN eine gegenüber PENCKs und EBERLs Beobachtungen beträchtlich nach Norden vorgeschobene Mindel-Riß-Grenze (vgl. dazu Abb. 6 und 7). GLÜCKERT und JERZ lassen diese Grenze nochmals um bis zu $2 \mathrm{~km}$ weiter nordwärts verlaufen, nach JERZ erreicht sie den Westrand des Böhener Feldes $1 \mathrm{~km}$ nördlich Schrattenbach. Dies steht aber in offenbarem Gegensatz zu den Verhältnissen in dem unmittelbar westlich benachbarten Talzug aus dem Dietmannsrieder Becken ins Memminger Trockental (vgl. dazu Abb. 1 u. 2). Hier hat es - ausweislich des Zeller Hochterrassensporns - eine Zunge des rißzeitlichen Gletschers gegeben, deren Ablagerungen, später vom Würm-Gletscher vollkommen überfahren wurden, die also nicht so weit reichte wie dieser. Zu diesen begrabenen Äußeren Riß-Endmoränen ergibt sich von dem 120 m höher liegenden Ausstrich der JERZschen Riß-Außengrenze nördlich Schrattenbach ein Gradient der Gletscheroberfläche von einer Steilheit, der physikalisch ganz unmöglich ist. Darauf hat bereits STEPP (1981: 56) deutlich hingewiesen. Noch problematischer - weil noch steiler — ist die Verbindung der JERZschen Mindel-Außengrenze mit den PENCKschen Mindel-Moränen an deren Typlokalität an der Wurzel des Grönenbacher Feldes (vgl. dazu Abb. 4).

Damit ist gezeigt, daß die Mindel-Riß-Grenze und auch die den Endmoränen von Brandholz-Waldegg entsprechende Mindel-Außengrenze auf dem Böhener Feld ganz sicher nicht da verlaufen, wo sie JERZ eingetragen hat. Wenn man diese Grenzen bestimmen will, muß man methodisch neu ansetzen, und zwar dort, wo ein einwandfreier Kontakt von Endmoränen und zugehörigen Schottern gegeben ist.

Für die Riß-Außengrenze ist das nur westlich der Iller beiderseits der würmzeitlichen Legauer Rinnen der Fall, wo Riß-Endmoränen den Ausläufern des Hitzenhofener Felds aufsitzen (vgl. Abb. 1). Allein von hier 


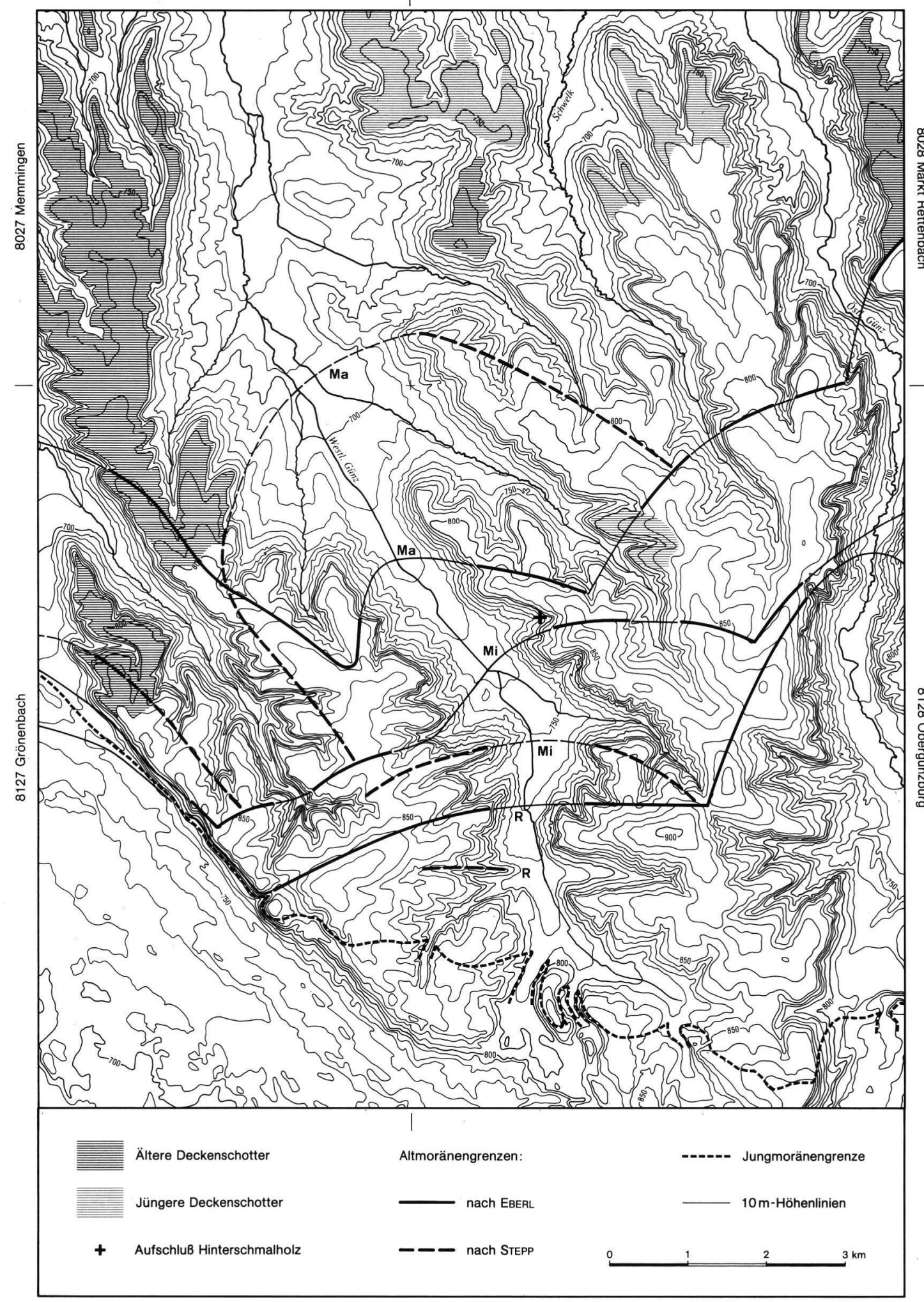

Abb. 6: Altmoränengliederung zwischen Memminger Trockental und Östlicher Günz nach EBERL 1930 und STEPP 1981. $\mathrm{Ma}=$ Äußere Moränenrandlage der Mindel-Eiszeit, $\mathrm{Mi}=$ Innere Moränenrandlage der Mindel-Eiszeit, 
aus kann man versuchen, die Riß-Grenze auch weiter im E zu bestimmen. Setzt man voraus, daß die Loben des rißzeitlichen Iller-Gletschers ein Längsgefälle ähnlich dem des - gut bekannten - würmzeitlichen gehabt haben, dann ergibt sich, daß die Riß-Außengrenze zwischen Iller und Böhener Feld allenfalls bei Herbistied auf eine kurze Strecke zutage ausstreicht, im übrigen aber beiderseits des Dietmannsrieder Zweigbeckens unter den würmzeitlichen $\mathrm{Ab}$ lagerungen verborgen bleibt. Östlich des Dietmannsrieder Zweigbeckens (vgl. dazu Abb. 8) taucht sie unter den Würm-Moränen erst am Südhang des Schellenbergs wieder auf - so wie das auch ELLWANGER (1980: 114, Abb. 1) und STEPP (1981: 58) schon gesehen haben - und setzt sich dann um den Simmerberg herum gegen Obergünzburg etwa so fort, wie das bereits EBERL (1930: 80 und Tafel I) angenommen hatte. Das heißt aber, daß der Hinterschmalholzer Boden weit außerhalb der Reichweite des rißzeitlichen Gletschers liegt. Es bezeichnet also nicht das Mindel-Riß-Interglazial.

In ähnlicher Weise kann man bei der Bestimmung der Mindel-Außengrenze verfahren. Hier ist von den PENCKschen Mindel-Endmoränen an der Wurzel des Grönenbacher Feldes auszugehen. Sie lassen sich sinnvoll nur mit den flachen Moränenrücken am Rande des Böhener Feldes oberhalb Schrattenbach verbinden, an deren Nordrand JERZ den Ausstrich seiner Riß-Außengrenze vermutete (vgl. Abb. 5). Schon EBERL (1930: 79 und Tafel I) hat das so gesehen und beide Moränen seinem Mindel I zugeordnet. Die Fortsetzung dieser Mindel-Außengrenze ist aber nicht wie bei EBERL vor dem Nordrand des Schellenberger Waldes zu suchen, weil der Schellenberg für den MindelGletscher - ähnlich wie für den jüngeren RißGletscher - ein wohl kaum überwindliches Hindernis dargestellt haben muß. Die Mindel-Außengrenze dürfte sich vielmehr - den heutigen Reliefverhältnissen entsprechend - zwar nördlich Bärenwies gegen die Ausläufer des Wolfertschwender Mühlbachtals vorgebuchtet haben, ist aber im übrigen am Innenrand des Schellenbergs verlaufen, um sich dann am Außenrand der etwas stärker reliefierten Moränenhöhen im Wasserscheidenbereich der beiden Günztäler fortzusetzen (vgl. Abb. 8). Westlich des Westlichen Günztals ist das der Rücken von Unter- und Oberwarlins, östlich davon jener von Hinterschmalholz. Beide zusammen markieren eine Zunge des Gletschers über dem Westlichen Günztal, die sich mit deutlich einspringendem Knick von den das Östliche Günztal begleitenden Moränenrücken absetzt, die die Verbindung zu den altbekannten Mindel-Moränen der Holzheuer Höhe oberhalb Ronsberg herstellen. Beiderseits des Westlichen Günztals hatte SINN (1972: Karte 5, vgl. Abb. 7) ganz ähnlich seine Mindel-RißGrenze gezeichnet.
Der einspringende Zwickel zwischen den beiden Loben über Westlichem und Östlichem Günztal ist vermutlich durch die hochaufragenden Höhen im Wasserscheidenbereich zwischen den beiden Günztälern (P. 901 ostwärts Simmerberg, P. 884 nordwestlich Hartmannsberg) bedingt. Bewiesen wird er durch die schon von SINN (1972: 117) erwähnten, von STEPP (1981: 59) näher beschriebenen Bremberg-Schotter, die im Moosmühlbach-Tal nördlich und östlich der Moosmühle liegen. SinN hat sie mit seinen Hangendmoränen von Hinterschmalholz verknüpft, sie also als Hochterrassenschotter angesehen. Für eine Hochterrasse liegt der Bremberg-Schotter aber viel zu hoch. Er ist daher von STEPP sicher mit Recht als Jüngerer Deckenschotter angesprochen worden. Er ist mithin ein Äquivalent des Grönenbacher Feldes und stützt damit die hier skizzierte Mindel-Außengrenze.

Außerhalb der so bestimmten Außengrenze der Mindel-Vereisung sensu PENCK verbleibt im Bereich zwischen Memminger Trockental und Östlichem Günztal ein Gebiet wenig reliefierter Altmoränen, die als flache Decke teils Tertiär, teils Ältere Deckenschotter überlagern. Ihre Außengrenze ist zwar umstritten (vgl. dazu Abb. 6 und 7), an ihrer Existenz besteht jedoch kein Zweifel. EBERL hat diese ältesten Altmoränen als Mindel II ausgegliedert, STEPP sie als äußere Hauptrandlagen (der Mindel-Moränen) bezeichnet. In Anlehnung daran könnte man sie als „Älteres Mindel" klassifizieren. Die im S angrenzenden Endmoränen des ,klassischen” Mindel sensu PENCK überlagern aber nicht nur die Moränen des „Älteren Mindel", sondern auch den Hinterschmalholzer Boden, der mit großer Wahrscheinlichkeit eine langdauernde Warmzeit, also ein Interglazial anzeigt. Das verbietet eigentlich die Bezeichnung von hangendem und liegendem Moränenkomplex mit dem gleichen Begriff. Andererseits sind auch die äußersten Altmoränen wegen ihrer breitflächigen Überlagerung der Älteren Deckenschotter - sicher jünger als Günz.

Nun haben SCHrEINER \& EBEL (1981) in dem dem Illergletscher-Gebiet westlich benachbarten östlichen Rheingletscher-Gebiet sichere Anzeichen einer zwischen Mindel- und Günz-Eiszeit einzuschiebenden - weiteren Eiszeit nachgewiesen, die sie als Haslach-Eiszeit bezeichnen. Die Abtrennung der Haslach-Eiszeit gegenüber Mindel und Günz ist dort zwar bisher nur in Bohrungen gelungen, sie ist aber u. a. - durch interglaziale Bodenbildungen gut belegt. Diese Mindel- und Haslach-Eiszeit trennenden Böden weisen nach Mächtigkeit und Bodeneigenschaften sehr viele Ähnlichkeiten mit dem Hinterschmalholzer Boden auf. Es liegt daher nahe, beides als gleichzeitige Bildungen einzustufen. Das würde aber bedeuten, daß die äußeren Altmoränen des Böhener Feldes der Haslach-Eiszeit zuzuweisen wären. 


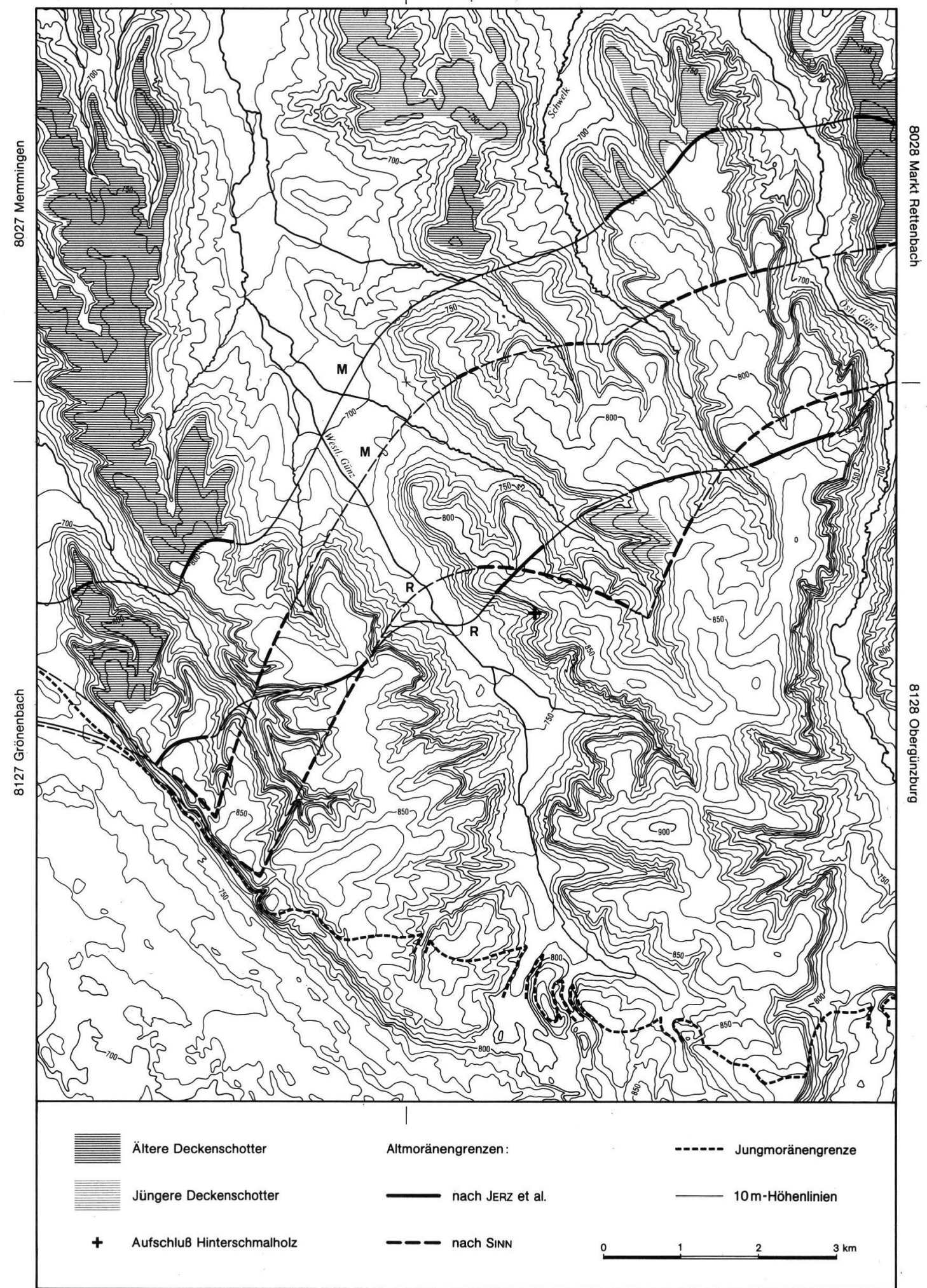

Abb. 7: Altmoränengliederung zwischen Memminger Trockental und Östlicher Günz nach SiNN 1972 und JeRZ et al. 1975. $\mathrm{M}=$ Außengrenze der Mindel-Altmoränen, $\mathrm{R}=$ Außengrenze der Riß-Altmoränen. 


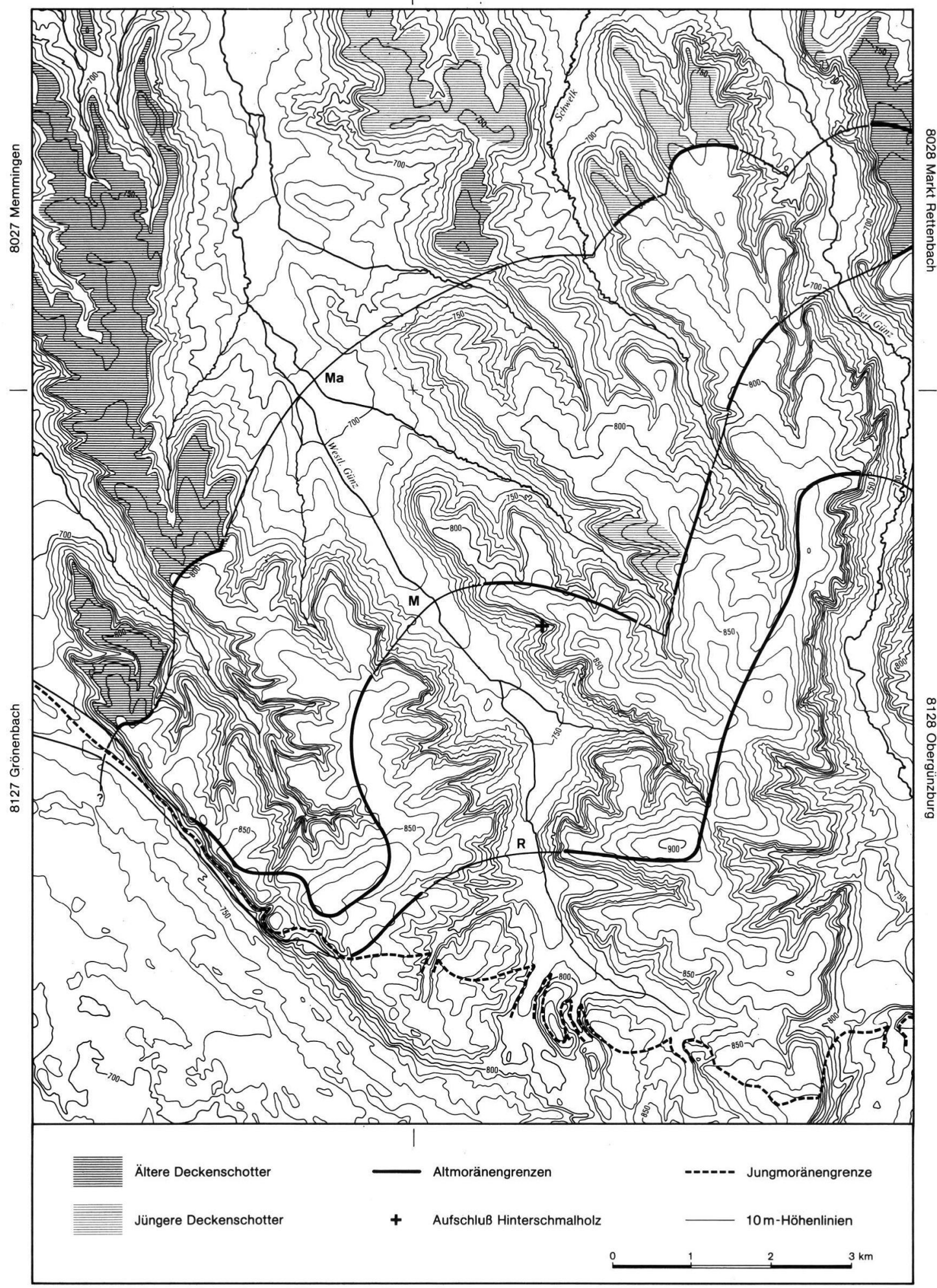

Abb. 8: Altmoränengliederung zwischen Memminger Trockental und Östlicher Günz nach eigenen Beobachtungen.

$\mathrm{Ma}=$ Außengrenze der Äußersten Altmoränen („Älteres Mindel” = „Haslach”?), M = Außengrenze der Mindel-Alt-Moränen sensu PENCK 1901 („Klassisches Mindel”), R = Außengrenze der Riß-Altmoränen. 
Dies ist freilich noch zu überprüfen. Es wäre unter anderem zu erklären, weshalb die der Haslach-Eiszeit zugeordneten Moränen in dem hier besprochenen Gebiet breitflächig an der Oberfläche liegen, während sie im östlichen Rheingletscher-Gebiet, wo sie zuerst nachgewiesen wurden, nur durch Bohrungen erschlossen, d. h. unter jüngeren Ablagerungen begraben sind. Doch gehört das schon nicht mehr zu den Arbeitsergebnissen, über die hier zu berichten war. Nicht zu bezweifeln ist aber, daß auch im Illergletscher-Gebiet zwischen klassischem Mindel sensu PENCK und Günz Ablagerungen einer weiteren Eiszeit vorhanden sind. Das wird bei zukünftigen Untersuchungen im Bereich der Iller-Lech-Platte zu beachten sein.

\section{Schriftenverzeichnis}

EBERL, B. (1930): Die Eiszeitenfolge im nördlichen Alpenvorlande - Ihr Ablauf, ihre Chronologie auf Grund der Aufnahmen des Lech- und Illergletschers. - 427 S.; Augsburg (Filser)

EICHLER, H. \& SinN, P. (1975): Zur Definition des Begriffs "Mindel" im schwäbischen Alpenvorland. - N. Jb. Geol. Paläontol. Mh. 1975/12: 705-718; Stuttgart.

ELLWANGER, D. (1980): Rückzugsphasen des würmzeitlichen Illergletschers. - Arb. Inst. Geol. Paläontol. Univ. Stuttgart, N. F., 76: 93-126; Stuttgart.

GLÜCKERT, G. (1974): Mindel- und rißzeitliche Endmoränen des Illervorlandgletschers. - Eiszeitalter u. Gegenwart, 25: 96-106; Öhringen.

Graul, H. \& Schaefer, I. (1953): Zur Gliederung der Würmeiszeit im Illergebiet. - Geologica Bavarica, 18: 5-112; München.

HAAG, TH. (1981): Tal- und flächenformende periglaziale und glaziale Dynamik im Glazialgebiet Süddeutschlands (Rheingletscher). - Bochumer Geogr. Arb., 40: 48-58; Paderborn.

- (1982): Das Mindelglazial des nordöstlichen Rheingletschergebietes zwischen Riß und Iller. - Jb. Mitteil. Oberrh. Geolog. Ver., 64: 225-266; Stuttgart.

Habbe, K. A. (1985a): Das Späthochglazial der WürmEiszeit im Illergletscher-Gebiet - Ergebnisse einer geomorphologischen Kartierung. - Quartär, 35/36: 55-68; Bonn.

- (1985b): Erläuterungen zur Geomorphologischen Karte 1:25000 der Bundesrepublik Deutschland GMK 25 Blatt 18, 8127 Grönenbach. - 83 S.; Berlin (GMK Schwerpunktprogramm).

Jerz, H., Stephan, W., Streit, R. \& Weinig, H. (1975): Zur Geologie des Iller-Mindel-Gebietes. - Geologica Bavarica, 74: 99-130; München.
LÖSCHER, M. (1976): Die präwürmzeitlichen Schotterablagerungen in der nördlichen Iller-Lech-Platte. - Heidelberger Geograph. Arb., 45: 157 S.; Heidelberg.

PENCK, A. \& BRÜCKNeR, E. (1901/09): Die Alpen im Eiszeitalter. - 3 Bd., 1199 S.; Leipzig (Tauchnitz).

RÖGNER, K. (1979): Die glaziale und fluvioglaziale Dynamik im östlichen Lechgletschervorland - Ein Beitrag zur präwürmzeitlichen Pleistozänstratigraphie. - Heidelberger Geograph. Arb., 49: 67-138; Heidelberg.

- (1980): Die pleistozänen Schotter und Moränen zwischen oberem Mindel- und Wertachtal (BayerischSchwaben). - Eiszeitalter u. Gegenwart, 30: 125-144; Hannover.

- (1981): Quartärmorphologische und -stratigraphische Probleme im schwäbisch-bayerischen Alpenvorland aufgezeigt an Beispielen der „Höhen über Kaufbeuren” und des "Warmisrieder Feldes”. - Bochumer Geograph. Arb., 40: 72-83; Paderborn.

SCHAEFER, I. (1940): Die Würmeiszeit im Alpenvorland zwischen Riß und Günz. - Abhandl. Naturkde.- u. Tiergartenverein Schwaben, 2: 148 S.; Augsburg.

- (1950): Die diluviale Erosion und Akkumulation Erkenntnisse aus Untersuchungen über die Talbildung im Alpenvorlande. - Forsch. dt. Landeskde, 49: 154 S.; Landshut.

- (1973): Das Grönenbacher Feld - Ein Beispiel für Wandel und Fortschritt der Eiszeitforschung seit Albrecht Penck. - Eiszeitalter u. Gegenwart, 23/24: 168-200; Öhringen.

- (1981): Die Glaziale Serie - Gedanken zum Kernstück der alpinen Eiszeitforschung. - Zeitschr. Geomorph. N. F., 25: 271-289; Berlin/Stuttgart.

- \& STePp, R. (1984): Zum Quartär in der Gegend von Obergünzburg. - Mitteil. Geograph. Gesellsch. München, 69: 195-205; München.

SCHrEINER, A. (1980): Zur Quartärgeologie in der Umgebung des Eem-Interglazials von Krumbach/Saulgau (Baden-Württemberg). - Geol. Jb., A 56: 5-43; Hannover.

- \& EBEL, R. (1981): Quartärgeologische Untersuchungen in der Umgebung von Interglazialvorkommen im östlichen Rheingletschergebiet (Baden-Württemberg). - Geol. Jb., A 59: 3-64; Hannover.

SINN, P. (1972): Zur Stratigraphie und Paläogeographie des Präwürm im mittleren und südlichen IllergletscherVorland. - Heidelberger Geograph. Arb., 37: 159 S.; Heidelberg.

STEPP, R. (1981): Das Böhener Feld - Ein Beitrag zum Altquartär im Südwesten der Iller-Lech-Platte. - Mitteil. Geograph. Gesellsch. München, 66: 43-68; München. 


\section{Geologische Karten}

Geologische Übersichtskarte des Iller-Mindel-Gebietes $1: 100000$. — Bearb. v. R. StReit, H. WeINIG, H. JeRZ \& W. Stephan. Hrsg. v. Bayer. Geolog. Landesamt. München 1975.

Geologische Übersichtskarte 1:200000 - Blatt CC 8726 Kempten (Allgäu). - Hrsg. v. d. Bundesanstalt für Geowissenschaften und Rohstoffe. Wissensch. Bearbeitung: H. SCHOLZ \& W. ZACHER. Hannover 1983.

\section{Topographische Karten}

Topographische Karte 1:25000 - Normalausgabe (N), Blätter 8027 Memmingen, 8028 Markt Rettenbach, 8127 Grönenbach, 8128 Obergünzburg.

Topographische Karte 1:50000 - Orohydrographische Ausgabe (OH), Blätter L 8126 Memmingen, L 8128 Kaufbeuren, L 8326 Kempten, L 8328 Marktoberdorf.

Manuskript eingegangen am 7. 1. 1986,

Nachträge März 1986. 